\title{
Bifurcations and feedback control of a stage-structure exploited prey- predator system
}

\author{
T. K. Kar* Bapan Ghosh \\ Department of Mathematics, Bengal Engineering and Science University, Shibpur, Howrah-711103, INDIA \\ "Corresponding Author: tkar1117@gmail.com
}

\begin{abstract}
The present paper describes a bioeconomic modelling of a stage-structure prey-predator system with differential algebraic equations. The criterion for coexistence of the equilibrium points and their stability nature are investigated. Singularity induced bifurcation are studied for zero economic profit and in this perspective, feedback control is designed to preserve the persistence property of the system. In contrast to zero profit, an interior equilibrium point remains stable for positive economic profit. The reasons behind the different nature of the interior equilibriums for zero and positive profit are discussed in conclusion section. Some numerical simulations are given to verify the analytical results. How the maximum profit hampers the system is provided through saddle-node bifurcation in the last subsection of numerical simulation.
\end{abstract}

Keywords: Prey-predator, stage-structure, singularity induced bifurcation, feedback control.

\section{Introduction and model description}

A major current focus related to the interacting prey-predator bioeconomic systems in presence of harvesting effort is to investigate the dynamical behaviour of the ecosystem towards the positive economic interest. Biological resource of prey-predator system is recently harvested unscientifically and exported with the aim of positive economic profit which gradually shortages the resources and the ecosystem is collapsed eventually. Nowadays, few research articles have proposed some harvesting strategies and management policies for long run biological resources. Idels and Wang (2008) have investigated the consequences of various harvesting strategies in single species fish population. Kar and Pahari (2007), Xiao et al. (2006) and Kumar et al. (2002) have studied the prey-predator model with harvesting and observed various complexity of the system namely, Bogdanov-Takens bifurcation, Hopf bifurcation, limit cycle, heteroclinic bifurcation and so on. Mazoudi et al. (2008) have considered age-structure fishery model and a Liapunav function is adapted to study the stability and stabilization of the system around the non-trivial steady states.

We shall now discuss some research articles which studied the dynamical behaviour of the bioeconomic model systems governed by some first order ordinary differential equations together with few algebraic equations; such systems are called differential algebraic equations (DAEs) systems. Zhang et al. (2009) have taken a differential algebraic prey-predator system with time delay where predator population is harvested continuously. They analyzed the transcritical bifurcation at a boundary point, singularity induced bifurcation at the unique singular point with respect to economic profit and well known Hopf bifurcation regarding the time delay parameter. Kar and Chakraborty (2010) have discussed with the same bioeconomic model harvesting the prey populations and removed completely the singularity induced bifurcation as well as the instability behaviour towards the positive economic profit by means of feedback control theory (Dai L. R., 1989). Zhang and Zhang (2009) modeled a differential algebraic equation system with a single harvesting population equation and a single algebraic equation, and the optimal control strategy is applied to eliminate the singularity induced bifurcation and minimize the cost energy on zero economic profit case. Liu et al. $(2008,2009)$ have constructed a harvested differential algebraic prey predator system and they demonstrated that the system is unstable for any positive economic interest (profit not very closed to zero) due to singularity induced bifurcation theory. But our recent model does not agree with the same. In such situations, the singularity induced bifurcation theory is not well fitted to describe the stabilization of the equilibrium points of the system. 
Here, we have considered a stage structure prey-predator model with stage structure for predator which is organized as follows:

$$
\begin{aligned}
& \frac{d N}{d \tau}=r_{1} N\left(1-\frac{N}{K}\right)-\alpha N N_{2}, \\
& \frac{d N_{1}}{d \tau}=\beta N_{2}-r_{2} N_{1}, \\
& \frac{d N_{2}}{d \tau}=r_{3} N_{2}+m \alpha N N_{2}+\gamma N_{1}-\delta N_{2}^{2},
\end{aligned}
$$

where $r_{1}$ is the intrinsic growth rate of prey population $N, K$ is the environmental carrying capacity for the prey, $\alpha$ is the predation rate of the mature predator $N_{2}$ over the prey $N, \beta$ is the transition rate from mature predator population $N_{2}$ to immature predator population $N_{1}, r_{2}$ and $r_{3}$ are the natural death rates of the immature and mature predator population respectively, $m$ measures how many portion of biomass is added to the mature predator population after predation, $\gamma$ is the conversion rate from immature to mature predator, $\delta$ is the coefficient of intraspecific competition of the mature predator and $\tau$ is the current time. All the biological meaningful parameters are positive.

We take the transformation for the state and time variable as follows: $N=K r_{2} x / r_{1}, \quad N_{1}=\beta y /(m \alpha), \quad N_{2}=r_{2} z /(m \alpha)$ and $\tau=t / r_{2}$, then the system (1) is converted to

$$
\begin{aligned}
& \dot{x}=a x-x^{2}-b x z, \\
& \dot{y}=z-y, \\
& \dot{z}=-c_{1} z+d x z+e y-s z^{2},
\end{aligned}
$$

where $a=r_{1} / r_{2}, b=1 / m, c_{1}=r_{3} / r_{2}, d=m \alpha K / r_{1}, e=\gamma \beta / r_{2}^{2}, s=\delta /(m \alpha)$ and $\dot{x}, \dot{y}, \dot{z}$ represent the derivatives of $x, y, z$ with respect to $t$ respectively.

Using the phase catch-per-unit-effort (CPUE) hypothesis (Clark, 1990) to describe an assumption that catch per unit effort is proportional to stock level we take the harvested term on mature predator as $H=q E z$, where $E$ is the harvesting effort and $q$ is the catchability co-efficient of the mature predator. Simultaneously an algebraic equation is also developed by considering the economic interest of harvesting according to Gordon's economic theory of a common property resource (Gordon, 1954). He established the economic interest of the yield of harvest effort as:

$$
\text { Net Economic Revenue }(N E R)=\text { Total Revenue }(T R)-\text { Total Cost }(T C) \text {. }
$$

In our present problem we suppose $T R=p q E z$ and $T C=c E$, where $p$ and $c$ respectively stand for the constant price per unit harvested biomass and constant harvesting cost per unit effort. Let us assume that $v$ is the NER, then the algebraic equation looks like

$$
(p q z-c) E=v
$$

Finally, the differential algebraic model system with harvesting predator takes the form

$$
\begin{aligned}
& \dot{x}=a x-x^{2}-b x z, \\
& \dot{y}=z-y, \\
& \dot{z}=-c_{1} z+d x z+e y-s z^{2}-q E z, \\
& 0=(p q z-c) E-v \text {. } \\
& \text { We now set } f:=\left(\begin{array}{l}
f_{1}(x, y, z, \mu) \\
f_{2}(x, y, z, \mu) \\
f_{3}(x, y, z, \mu)
\end{array}\right)=\left(\begin{array}{c}
a x-x^{2}-b x z \\
z-y \\
-c_{1} z+d x z+e y-s z^{2}-q E z
\end{array}\right) \text {, }
\end{aligned}
$$


$g(x, y, z, \mu)=(p q z-c) E-v$ and $X=[x, y, z]$ is a three dimensional column vector.

The matrix representation of equation (5) is ultimately expressed as $A\left(\begin{array}{l}\dot{X} \\ 0\end{array}\right)=\left(\begin{array}{l}f \\ g\end{array}\right)$,

where $A=\left(\begin{array}{cccc}1 & 0 & 0 & 0 \\ 0 & 1 & 0 & 0 \\ 0 & 0 & 1 & 0 \\ 0 & 0 & 0 & 0\end{array}\right)$ is a singular matrix.

\section{Equilibria and their stability analysis in zero economic profit}

When economic profit is zero then the model system (5) reduces to

$$
\begin{aligned}
& \dot{x}=a x-x^{2}-b x z, \\
& \dot{y}=z-y, \\
& \dot{z}=-c_{1} z+d x z+e y-s z^{2}-q E z, \\
& 0=(p q z-c) E .
\end{aligned}
$$

The above system always possesses two equilibrium points $P_{0}(0,0,0,0)$ and $P_{1}(a, 0,0,0)$. The boundary equilibrium point $P_{2}\left(x_{2}, y_{2}, z_{2}, E_{2}\right)$ and the interior equilibrium point $P_{*}\left(x_{*}, y_{*}, z_{*}, E_{*}\right)$ exist under some considerations, where $x_{2}=\left(a s+b\left(c_{1}-e\right)\right) /(b d+s), \quad y_{2}=\left(a d+e-c_{1}\right) /(b d+s), z_{2}=\left(a d+e-c_{1}\right) /(b d+s), \quad E_{2}=0 ; \quad$ and $x_{*}=(a p q-b c) / p q, \quad y_{*}=c / p q$, $Z_{*}=c / p q, \quad E_{*}=\left(p q\left(a d+e-c_{1}\right)-c(b d+s)\right) / p q^{2}$. If $a p q>b c$ and $p q\left(a d+e-c_{1}\right)>c(b d+s)$, then $P_{*}$ exists. The existence of $P_{*}$ ensures the existence of $P_{2}$ together with the condition $\left(a s+b c_{1}\right)>b e$. But, if $P_{2}$ does not exit yet $\left(a s+b c_{1}\right)>b e$, then $P_{*}$ never exists. Thus both the equilibrium exist when $\left(a s+b c_{1}\right)>b e, a p q>b c$ and $p q\left(a d+e-c_{1}\right)>c(b d+s)$. Out of these $P_{*}$ is the only interior singular equilibrium (definition would be lunched shortly) point. The interior nature of $P_{*}$ prevents $P_{2}$ not to be singular which follows from $E_{*}>0$ i.e. $x_{2}>x_{*}$. To describe the stability of different equilibrium points we evaluate the Jacobian matrix $J_{3}$ of the system (7) at an arbitrary point is defined in a special form as

$$
\begin{aligned}
J_{3} & =D_{X} f-D_{E} f\left(D_{E} g\right)^{-1} D_{X} g \\
& =\left(\begin{array}{ccc}
-x & 0 & -b x \\
0 & -1 & 1 \\
z d & e & -c_{1}+x d-2 s z-q E+\frac{p q^{2} E z}{(p q z-c)}
\end{array}\right) .
\end{aligned}
$$

First of all we shall discuss the stability analysis of the three equilibrium points viz., $P_{0}, P_{1}$ and $P_{2}$ by calculating the eigen values of the Jacobian matrix $J_{3}$ at the corresponding points.

At $P_{0}$, one eigen value of the community matrix is $a$. Consequently $P_{0}$ is an unstable node or unstable focus. Also $-a$ is an eigen value at $P_{1}$ of the linearised system (7), hence the stability nature can be completely determined by the solutions of the equation

$$
\lambda^{2}+\left(1+c_{1}-a d\right) \lambda+\left(c_{1}-a d-e\right)=0 .
$$


Theorem 2.1 The equilibrium point $P_{2}$ is stable for all biological parameters.

Proof. The characteristic equation of the jacobian matrix at $P_{2}$ is

$$
\begin{gathered}
\tau^{3}+w_{1} \tau^{2}+w_{2} \tau+w_{3}=0, \text { where } \\
w_{1}=e_{1} y_{2} / z_{2}+s z_{2}+x_{2}+1, \quad w_{2}=\left(x_{2}+1\right)\left(e y_{2} / z_{2}+s z_{2}\right)+b d x_{2} z_{2}+x_{2}-e \quad \text { and } \quad w_{3}=x_{2}\left[\left(e y_{2} / z_{2}+s z_{2}\right)+b d z_{2}-e\right] .
\end{gathered}
$$

It is easy to follow that $w_{1}>1, \quad w_{3}>0$ and $w_{2}-w_{3}=(1+e) x_{2}+s z_{2}$. Therefore, $w_{1} w_{2}-w_{3}>0$ for any sets of biological parameters. Hence by Routh-Hurwitz criterion, $P_{2}$ is a stable equilibrium point.

Theorem 2.2 When the economic profit is negative and closed to zero, the system (7) is stable at $P_{*}$ for any set of meaningful biological parameters.

Proof. Let us assume that $m_{11}, m_{22}$, and $m_{33}$ are the principal diagonal minors of the community matrix at $P_{*}$ for arbitrary small $v<0$. Then $m_{11}=-x_{*}<0, m_{22}=x_{*}>0$ and

$$
m_{33}=X_{*} Z_{*}\left(\frac{p q^{2} E_{*}}{p q Z_{*}-c}-(b d+s)\right)<0 .
$$

So we can demonstrate that all the eigen values corresponding to the system (7) lie in $C^{-}$(left half complex plane). Hence $P_{*}$ is stable for very small negative economic profit.

\subsection{Singular induced bifurcation in a differential algebraic equations (DAEs) system}

The DAEs system can be put in the form

$$
\begin{aligned}
& \dot{x}=f(x, y, \mu), \quad f: \Re^{n+m+r} \rightarrow \Re^{n}, \\
& 0=g(x, y, \mu), \quad g: \Re^{n+m+r} \rightarrow \Re^{m},
\end{aligned}
$$

where $x \in \Theta \subset \mathfrak{R}^{n}, y \in \Omega \subset \mathfrak{R}^{m}, \mu \in \Lambda \subset \mathfrak{R}^{r}$ with $n, m$ and $r$ are all positive integers. In this particular section, $x$ is the dynamic state vector whose time evaluation is directly connected by the equation (9a) and $y$ is the instantaneous state vector which satisfies the constraint equation (9b) and the parameter set $\mu$ defines a specific system configuration and operating condition.

We define the set of all equilibria of the DAEs system (9a)-(9b) to be EQ and the set of all stable equilibria $O P$ as

$$
\begin{aligned}
& E Q=\{(x, y, \mu) \in \Theta \times \Omega \times \Lambda: f(x, y, \mu)=0, g(x, y, \mu)=0\} \text { and } \\
& O P=\left\{(x, y, \mu) \in E Q: \operatorname{Det}\left(D_{y} g\right) \neq 0, \operatorname{Re}\left(\lambda\left(J_{n}\right)\right)<0\right\},
\end{aligned}
$$

where $\lambda\left(J_{n}\right)$ is the set of all eigen values corresponding to the Jacobian matrix $J_{n}=D_{x}-D_{y} f\left(D_{y} g\right)^{-1} D_{x} g$ of the system (9a)(9b). We also define the singular surface

$$
S=\left\{(x, y, \mu) \in \Theta \times \Omega \times \Lambda: g(x, y, \mu)=0, \Delta(x, y, \mu):=\operatorname{Det}\left(D_{y} g\right)=0\right\}
$$

and corresponding point on $S$ is known as singular point which plays an important role in differential algebraic system. In a DAEs system the singularity induced bifurcation (SIB) occurs if equilibrium crosses the singular surface $S$ at bifurcation point. Trajectories cross the singularity in a finite time with an infinite speed and the system changes its stability due to an eigen value diverging to infinity. This type of bifurcation can be analyzed with the help of the following theorem.

\section{Theorem 2.3 (Singularity induced bifurcation theorem)}


Suppose the system (9a)-(9b) satisfies the following conditions at the singular equilibrium point $\left(x_{0}, y_{0}, \mu_{0}\right)$ :

SIB1: $D_{y} g$ has a simple zero eigen value and Trace $\left(D_{y} f\left(D_{y} g\right)^{-1} D_{x} g\right)$ is nonzero.

SIB2: $\quad\left(\begin{array}{cc}D_{x} f & D_{y} f \\ D_{x} g & D_{y} g\end{array}\right)$ is nonsingular.

SIB3: $\left(\begin{array}{ccc}D_{x} f & D_{y} f & D_{\mu} f \\ D_{x} g & D_{y} g & D_{\mu} g \\ D_{x} \Delta & D_{y} \Delta & D_{\mu} \Delta\end{array}\right)$ is also nonsingular.

Then according to Venkatasubramanian (1992) \& Venkatasubramanian et al. (1995), there exist a smooth curve of the equilibrium in $\mathfrak{R}^{n+m+r}$ which passes through $\left(x_{0}, y_{0}, \mu_{0}\right)$ and is transversal to the singular surface at $\left(x_{0}, y_{0}, \mu_{0}\right)$. When $\mu$ increases through $\mu_{0}$ one eigen value of the Jacobian matrix $J_{n}$ moves from $C^{-}$to $C^{+}$if $M / N>0$ (respectively from $C^{+}$to $C^{-}$if $\left.M / N<0\right)$ along the real axis by diverging through infinity. The rest $(n-1)$ eigen values remain bounded and stay away from the origin. The constants $M$ and $N$ can be computed by evaluating

$$
\begin{aligned}
& M=-\operatorname{Trace}\left(D_{y} f\left(\operatorname{adj}\left(D_{y} g\right)\right) D_{x} g\right) \text { and } \\
& N=D_{\mu} \Delta-\left(\begin{array}{ll}
D_{x} \Delta & D_{y} \Delta
\end{array}\right)\left(\begin{array}{ll}
D_{x} f & D_{y} f \\
D_{x} g & D_{y} g
\end{array}\right)^{-1}\left(\begin{array}{c}
D_{\mu} f \\
D_{\mu} g
\end{array}\right) .
\end{aligned}
$$

\subsection{Singularity induced bifurcation at $P_{*}$}

Theorem 2.4 Considering the positive meaningful biological parameters when the economic parameter $v$ increases through 0 , the system (7) undergoes singularity induced bifurcation at the equilibrium $P_{*}$ and the stability of the equilibrium point $P_{*}$ changes from stable to unstable.

Proof. SIB1: At $P_{*}, \Delta:=D_{E} g=p q z-c$ has a simple zero eigen value and

$$
\left.\operatorname{Trace}\left(D_{E} f\left(\operatorname{adj}\left(D_{E} g\right)\right) D_{X} g\right)\right|_{P_{*}}=-p q^{2} E_{*} Z_{*} \neq 0
$$

SIB2: $\quad\left|\begin{array}{ll}D_{X} f & D_{E} f \\ D_{X} g & D_{E} g\end{array}\right|_{P_{*}}=\left|\begin{array}{rrrc}-x & 0 & -b x & 0 \\ 0 & -1 & 1 & 0 \\ -z d & e & -e-s y & -q z \\ 0 & 0 & p q E & p q z-c\end{array}\right|_{P_{*}}=p q E_{*} Z_{*} \neq 0$ and

SIB3: $\left.\quad \begin{array}{lll}D_{X} f & D_{E} f & D_{\mu} f \\ D_{X} g & D_{E} g & D_{\mu} g \\ D_{X} \Delta & D_{E} \Delta & D_{\mu} \Delta\end{array}\right|_{P_{*}}=\left|\begin{array}{rrccc}-x & 0 & -b x & 0 & 0 \\ 0 & -1 & 1 & 0 & 0 \\ -z d & e & -e-s y & -q z & 0 \\ 0 & 0 & p q E & p q z-c & -1 \\ 0 & 0 & p q & 0 & 0\end{array}\right|_{P_{*}}=-p q^{2} E_{*} X_{*} \neq 0$.

Also $M=-\left.\operatorname{Trace}\left(D_{E} f\left(\operatorname{adj}\left(D_{E} g\right)\right) D_{X} g\right)\right|_{P_{*}}=p q^{2} E_{*} Z_{*}>0$ and 


$$
N=\left.D_{\mu} \Delta\right|_{P_{*}}-\left.\left(\begin{array}{cc}
D_{X} \Delta & D_{E} \Delta
\end{array}\right)\left(\begin{array}{cc}
D_{X} f & D_{E} f \\
D_{X} g & D_{E} g
\end{array}\right)^{-1}\left(\begin{array}{c}
D_{\mu} f \\
D_{\mu} g
\end{array}\right)\right|_{P_{*}}=\frac{1}{E_{*}}>0 .
$$

Finally, $\frac{M}{N}=p q^{2} E_{*}^{2} Z_{*}>0$.

According to the theorem 2.3, the system (7) undergoes SIB at the equilibrium $P_{*}$ when the bifurcation parameter $\mu:=v=0$. When $v$ increases through 0 , one eigen value of the system Jacobian $J_{3}$ at $P_{*}$ moves from $C^{-}$to $C^{+}$along the real axis and ultimately diverges to infinity as $v$ is highly closed to 0 . This eigen value approximately is equal to $\left(p q^{2} E_{*}^{2} Z_{*} / v-e-s Z_{*}\right)$ at the neighbourhood of $P_{*}$ (or equivalently at the neighbourhood of $v=0$ ). It brings an impulse in the said population system and the ecosystem collapsed very rapidly. The other two eigen values remain bounded and stay in $C^{-}$, away from the origin. Consequently the stability of model system (7) changes from stable to unstable at the equilibrium point $P_{*}$ when the economic profit increases through zero. Hence the proof is complete.

\section{Design of the feedback control}

In the case of zero economic profit the system (7) is unstable around the equilibrium point $P_{*}$. To eliminate the singularity induced bifurcation and stabilize the system (7), a state feedback control is designed under certain condition when economic profit $v=0$. In the next subsection, we also fit the same to stabilize an unstable equilibrium point $P^{*}\left(x^{*}, y^{*}, z^{*}, E^{*}\right)$ regarding to a suitable positive economic profit $v=v^{*}$.

The Jacobian matrix of (7) at an interior point can also be put in the form as follows:

$$
J_{4}=\left(\begin{array}{rrrc}
-x & 0 & -b x & 0 \\
0 & -1 & 1 & 0 \\
-z d & e & -e-s y & -q z \\
0 & 0 & p q E & p q z-c
\end{array}\right)
$$

Now $\operatorname{rank}\left(J_{4}, A J_{4}, A^{2} J_{4}, A^{3} J_{4}\right)_{P_{*}}=4$, where $A$ is described earlier. According to the Theorem 2-2.1 in Dai (1989), the model system (7) is locally controllable at $P_{*}$. In the status of the Theorem 3-1.2 in Dai (1989), a state feedback controller $u=k\left(E-E_{*}\right)$ can be applied to stabilized the differential algebraic system, where $E_{*}=\left(p q\left(a d+e-c_{1}\right)-c(b d+s)\right) / p q^{2}$ is the $E$ component of $P_{*}$ and $k$ is called the feedback gain. Hence the system (7) reduces to

$$
\begin{aligned}
& \dot{x}=a x-x^{2}-b x z, \\
& \dot{y}=z-y, \\
& \dot{z}=-c_{1} z+d x z+e y-s z^{2}-q E z, \\
& 0=(p q z-c) E+k\left(E-E_{*}\right) .
\end{aligned}
$$

Remark 3.1 At $P_{*}, J_{3}$ and $J_{4}$ both are the Jacobian matrices of the same model system (7), but of different orders and elements. Also $\operatorname{Det}\left(J_{3}\right) \neq \operatorname{Det}\left(J_{4}\right)$. The characteristic polynomial of $J_{3}$ follows the expansion of $\operatorname{Det}\left(I_{3} \lambda-J_{3}\right)$, but in the case of $J_{4}$ it is of $\operatorname{Det}\left(A \lambda-J_{4}\right)$, which is expected as the system (7) consists of three differential equations of first order and first degree. These two characteristic polynomials are different with respect to the coefficients of $\lambda$, but of same degree. The first one is a monic polynomial of third degree, where as the second one is not monic. Both the polynomials agree at their zeros. We use these according to our necessity.

Theorem 3.1 If the feedback gain $k$ satisfies either

$$
k>\operatorname{Max}\left\{p q^{2} E_{*} Z_{*} /\left(s Z_{*}+X_{*}+e+1\right), p q^{2} E_{*} /(b d+s)\right\} \text { or }
$$




$$
k>\operatorname{Max}\left\{p q^{2} E_{*} Z_{*} /\left(s Z_{*}+X_{*}+e+1\right), p q^{2} E_{*} /(b d+s), k_{*}\right\},
$$

where $k_{*}$ is the largest positive root of the equation

$$
k^{2}+\frac{B_{*}}{C_{*}} k+\frac{A_{*}}{C_{*}}=0
$$

then the system (11) is asymptotically stable at $P_{*}$.

$$
\text { Here } \begin{aligned}
A_{*} & =\left(x_{*}+1\right)\left(p q^{2} E_{*} Z_{*}\right)^{2}, \quad B_{*}=-p q^{2} E_{*} Z_{*}\left[\left(1+x_{*}^{2}\right)^{2}+2 X_{*}\left(e+s Z_{*}\right)+b d x_{*} Z_{*}+2 s Z_{*}+e\right] \text { and } \\
C_{*} & =\left[\left(\left(1+x_{*}+e+s Z_{*}\right)\left(1+x_{*}+x_{*}^{2}\right)+\left(1+X_{*}+e+s Z_{*}\right)\left(b d x_{*} Z_{*}+x_{*}-e\right)+\left(1+x_{*}\right)\left(e+s Z_{*}\right)^{2}+e X_{*}-b d x_{*} Z_{*}\right]\right. \\
& >e+b d x_{*} Z_{*}-e\left(1+x_{*}+e+s Z_{*}\right)+\left(e+s Z_{*}\right)^{2}+e X_{*}-b d x_{*} Z_{*} \\
& =\left(e+s Z_{*}\right)^{2}-e\left(e+s Z_{*}\right)>0 .
\end{aligned}
$$

Proof. The Jacobian matrix of the system (11) at $P_{*}$ is

$$
\left(\begin{array}{rrr}
-x & 0 & -b x \\
0 & -1 & 1 \\
z d & e & -e-s z+\frac{p q^{2} E z}{k}
\end{array}\right)_{P_{*}}
$$

Its characteristic equation is $\lambda^{3}+\sigma_{1} \lambda^{2}+\sigma_{2} \lambda+\sigma_{3}=0$, where $\sigma_{1}=1+x_{*}+e+s Z_{*}-p q^{2} E_{*} Z_{*} / k$, $\sigma_{2}=\left(x_{*}+1\right)\left(e+s Z_{*}-p q^{2} E_{*} Z_{*} / k\right)+b d x_{*} Z_{*}+x_{*}-e$ and $\sigma_{3}=x_{*}\left(s Z_{*}+b d Z_{*}-p q^{2} E_{*} Z_{*} / k\right)$.

Now $\sigma_{1}>0 \Rightarrow k>p q^{2} E_{*} Z_{*} /\left(1+x_{*}+e+s Z_{*}\right)$ and $\sigma_{3}>0 \Rightarrow k>p q^{2} E_{*} /(b d+s)$. Also $\sigma_{1} \sigma_{2}-\sigma_{3}=A_{*} / k^{2}+B_{*} / k+C_{*}$. For any set of biological parameters $A_{*}>0$ always holds. Now, if $B_{*}{ }^{2}-4 A_{*} C_{*}<0$, then the expression $\sigma_{1} \sigma_{2}-\sigma_{3}$ is always positive for any real value of $k$. Hence by Routh-Hurwitz criteria (Kot, 2001), the DAEs system (11) at $P_{*}$ is stable and the corresponding feedback gain $k$ satisfies

$$
k>\operatorname{Max}\left\{p q^{2} E_{*} Z_{*} /\left(s Z_{*}+X_{*}+e+1\right), p q^{2} E_{*} /(b d+s)\right\} .
$$

Otherwise, if $B_{*}{ }^{2}-4 A_{*} C_{*}<0$ does not hold, then the expression $\sigma_{1} \sigma_{2}-\sigma_{3}=\left(C_{*} / k^{2}\right)\left[k^{2}+\left(B_{*} / C_{*}\right) k+\left(A_{*} / C_{*}\right)\right]$ is greater than zero for any $k>k_{*}$. Consequently, the system is stable for

$$
k>\operatorname{Max}\left\{p q^{2} E_{*} Z_{*} /\left(s Z_{*}+x_{*}+e+1\right), p q^{2} E_{*} /(b d+s), k_{*}\right\} .
$$

Hence the proof

From a bioeconomic system, a society or the government of a country always expects some positive profit. In a real fishery management, the fishery agencies are interested towards the positive economic rent from the fishery. But, we can not expect a high economic profit in real life situation, as it hampers the persistent property of the ecosystem. Thus the economic profit runs over a suitable interval $\left(0, v_{e}\right)$. Suppose $v^{*}$ is our targeted positive profit over $\left(0, v_{e}\right)$, then it ensures at least one interior positive equilibrium point $P^{*}\left(x^{*}, y^{*}, z^{*}, E^{*}\right)$ of the model system and for $v^{*} \geq v_{e}$ the system has no positive equilibrium point at all. Liu et al. in $(2008,2009)$ have demonstrated that any positive equilibrium point corresponding to a positive profit in their bioecononic DAEs system is always unstable according to the singularity induced bifurcation theorem. But, no singular point exists for positive economic profit as $\operatorname{Det}\left(D_{E} g\right)=v / E>0$ and singularity induced bifurcation theory can not be permitted to apply for analyzing the stability nature of the equilibrium points. So, there may exist positive equilibrium point associated with a positive profit for which the model system (5) is stable in its own rights. Assume $P^{*}\left(x^{*}, y^{*}, z^{*}, E^{*}\right)$ is an unstable equilibrium point related to $v=v^{*}$ of the system (5), where $x^{*}=a-b z^{*}, y^{*}=z^{*}, E^{*}=v^{*} /\left(p q z^{*}-c\right)$ and $z^{*}$ is a typical solution of the equation

$$
p q(b d+s) z^{2}-\left[p q\left(a d+e-c_{1}\right)+c(b d+s)\right] z+\left[c\left(a d+e-c_{1}\right)+q v^{*}\right]=0 .
$$


Then we can, once again, design a feedback controller $u(t)=k\left(E-E^{*}\right)$, applying the same assumptions discussed in the front of this section. Adding the feedback controller into the model system (5) regarding to $v=v^{*}$, the reduced final model is

$$
\begin{aligned}
& \dot{x}=a x-x^{2}-b x z, \\
& \dot{y}=z-y, \\
& \dot{z}=-c_{1} z+d x z+e y-s z^{2}-q E z, \\
& 0=(p q z-c) E-v^{*}+k\left(E-E^{*}\right)
\end{aligned}
$$

and the associated Jacobian matrix is

$$
\left(\begin{array}{rrc}
-x & 0 & -b x \\
0 & -1 & 1 \\
z d & e & -e-s z+\frac{p q^{2} E z}{(p q z-c)+k}
\end{array}\right)_{P^{*}}
$$

We now calculate $k$ by means of numerical approach.

\section{Numerical simulation}

For verification of our previously discussed analytical results, we, here, would like to present some numerical simulations with the help of MATHEMATICA 5.2 and MATLAB 7.0 software packages.

(I) In this subsection, numerical support is provided for the singularity induced bifurcation with the hypothetical data as $a=5$, $b=0.5, c_{1}=15, d=2, e=10, s=0.05, p=20, q=0.2, c=15$ and $v$ is the bifurcation parameter. For zero economic profit of the model system (7), there exists unique singular equilibrium point $P_{*}(3.125,3.75,3.75,5.3125)$. Now the arbitrary variation of $v$ (very closed to zero) changes the local stability of $P_{*}$. The variation of $v$ and the corresponding eigen values are shown in the following Table 1 and together with the Figure $1 \&$ Figure 2 below.

Table 1. The variation of $v$ and the corresponding eigen values

\begin{tabular}{|l|l|l|l|}
\hline $\begin{array}{l}\text { Eigen values } \mapsto \\
v \downarrow\end{array}$ & $\operatorname{Real}\left(\lambda_{1}\right)$ & $\operatorname{Real}\left(\lambda_{2}\right)$ & $\operatorname{Real}\left(\lambda_{3}\right)$ \\
\hline-0.001 & -0.99999 & -3.1252 & -84684.97 \\
\hline-0.0001 & -0.99999 & -3.1250 & -846696.68 \\
\hline 0.001 & -1.00012 & -3.1248 & 84650.97 \\
\hline 0.0001 & -1.00001 & -3.1249 & 846662.69 \\
\hline
\end{tabular}




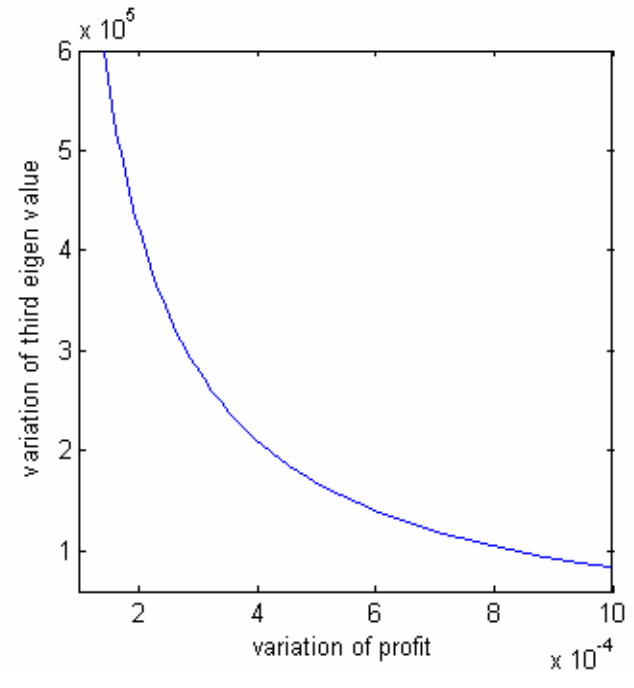

Figure 1. Variation of the third eigen value for positive $\mathrm{v}$ (very closed to zero).

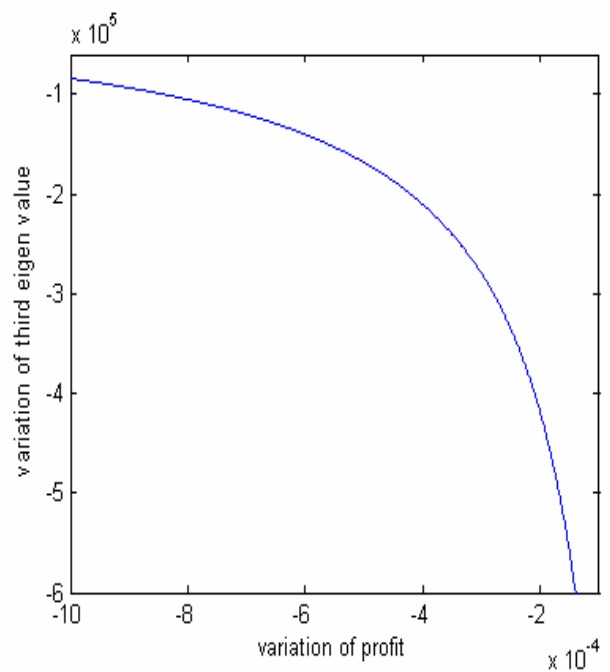

Figure 2. Variation of the third eigen value for negative $\mathrm{v}$ (very closed to zero).

(II) To eliminate the singularity induced bifurcation at $P_{*}(3.125,3.75,3.75,5.3125)$, we take the feedback gain $k=20$, which is greater than $\operatorname{Max}\{1.114,4.048\}$. Using this condition the system (11) possesses the eigen values as $-8.556,-4.716$ and -0.243 , consequently the system is stable at $P_{*}$.

(III) Numerically we can calculate that the maximum positive bioeconomic profit $v_{e} \approx 5.37574$. If we want to investigate the positive profit $v^{*}=3$, then there exists a typical equilibrium point $\hat{P}(2.7038,4.5923,4.5923,0.8904)$, which is stable without any feedback control and no singularity induced bifurcation occurs for positive profit (positive profit not very closed to zero). In contrast to the above fact, if we take $v^{*}=2.5$, then a typical equilibrium $P^{*}(3.0570,3.8859,3.8859,4.5990)$ exists which is unstable in nature. Applying feedback control, we have $k>\operatorname{Max}\{0.4044,3.8538\}$ and the eigen values of the system (14) are 7.5757, -6.3977 and -0.1869 corresponding to $k=15$.

(IV) In previous section, we have demonstrated that there exists no equilibrium point in the system when economic profit exceeds its maximum value. For example, if we consider the same set of biological parameters as in $(I)$, then the maximum profit $v_{e} \approx 5.37574$. In particular, there exist a stable equilibrium $P^{S}(2.8051,4.3897,4.3897,1.9540)$ and unstable equilibrium $P^{u}(2.9389,4.1222,4.1222,3.3585)$ corresponding to $v=5$. These two equilibrium approach to each other as $v$ increases and disappear when $v$ crosses $v_{e}$. Thus the maximum profit is a bifurcation parameter which compel the populations to be extinct forever. This phenomenon can be proved numerically through saddle-node bifurcation as follows:

When profit is positive all the equilibrium are nonsingular points. Therefore, applying the literature proposed by Venkatasubramanian et al. (1995), the differential algebraic system can be reduced to ordinary differential system as $\dot{X}=f_{R}(X, \mu)$ locally near any equilibrium point by a suitable (unique) function $f_{R}$. Now the new constructed system satisfies the following conditions near $P_{e}\left(x_{e}, y_{e}, z_{e}, E_{e}\right)=(2.8720,4.2559,4.2559,2.6562)$ corresponding to $v_{e}$.

SND-1: $\quad\left(D_{X} f-D_{E} f\left(D_{E} g\right)^{-1} D_{X} g\right)_{P_{e}}=\left(\begin{array}{ccc}-2.8720 & 0 & -1.4360 \\ 0 & -1 & 1 \\ 8.5119 & 10 & -5.7441\end{array}\right)$ has a simple zero eigen value with right vector 


$$
\vec{\Phi}=\left(\begin{array}{c}
\Phi_{1} \\
\Phi_{2} \\
\Phi_{3}
\end{array}\right)=\left(\begin{array}{c}
0.3333 \\
-0.6667 \\
-0.6667
\end{array}\right)
$$

and left eigen vector $\vec{\Psi}=\left(\begin{array}{lll}\Psi_{1} & \Psi_{2} & \Psi_{3}\end{array}\right)=\left(\begin{array}{lll}0.2827 & 0.9544 & 0.0954\end{array}\right)$,

SND-2: $\quad\left(\vec{\Psi} D_{\mu} f_{R}\right)_{P_{e}}=\left(\vec{\Psi}\left(D_{\mu} f-D_{E} f\left(D_{E} g\right)^{-1} D_{\mu} g\right)\right)_{P_{e}}=-\frac{q z_{e} \Psi_{3}}{p q z_{e}-c}=-0.04 \neq 0$ and

SND-3: $\quad\left(\vec{\Psi}\left(D_{X}^{2} f_{R}(\vec{\Phi}, \vec{\Phi})\right)\right)_{P_{e}}=\left(\vec{\Psi} \sum_{i=1}^{3}\left(e_{i} \vec{\Phi}^{\prime}\left(D_{X}\left(D_{X} f_{i}\right)^{\prime}\right) \vec{\Phi}\right)\right)_{P_{e}}$

$$
=-2\left[\Phi_{1} \Psi_{1}\left\{\Phi_{1}+b \Phi_{3}\right\}+\Phi_{3} \Psi_{3}\left\{\left(s+\frac{p q^{2} E_{e} c}{\left(p q z_{e}-c\right)^{2}}\right) \Phi_{3}-d \Phi_{1}\right\}\right]=-0.705 \neq 0,
$$

where $\vec{\Phi}^{\prime}$ stands for transpose of $\vec{\Phi}$ and so on.

According to the literature (Guckenheimer \& Holems, 1983), the system undergoes saddle node bifurcation at $P_{e}$.

\section{Conclusions}

The present paper deals with a stage structure prey-predator model with harvesting and it is proposed by means of a system of differential algebraic equations. Dynamical behaviour of the model is investigated due to the variation of the economic interest of harvesting. Singularity induced bifurcation and feedback control technique are studied. To stabilize the system at an interior point, the positive profit and the harvesting effort must satisfy the relation $E^{2} / v<(b d+s) / p q^{2}$ (from Theorem 2.2). For zero profit case the condition does not hold at all. In the last section of numerical simulation, $P^{s}$ satisfies the above relation, but $P^{u}$ does not. When $v$ increases, the $E$ component of $P^{s}$ increases and for the case of $P^{u}$, it decreases continuously. At a certain value of $v$, the relation reduces to $E^{2} / v \approx(b d+s) / p q^{2}$ and community matrix possesses a simple zero eigen value which causes the saddle-node bifurcation in the system. Therefore, in fishery management, fishery agency always should take care of it.

The model and its dynamical behaviour are studied mainly on the deterministic framework. It will be more realistic to consider the model in a stochastic environment due to either ecological or economic fluctuations. This may be considered in future work.

\section{Acknowledgement}

This research work is supported by the Council of Scientific and Industrial Research (CSIR), India (Grant No. 25(0160)/08/EMR-II dated 17.01.08).

\section{References}

Clark C. W., 1990. Mathematical Bioeconomics: The Optimal Management of Renewable Resources, $2^{\text {nd }}$ ed. John Wiley and Sons, New York.

Dai L. R., 1989. Singular Control System, Springer-Verlag.

Gordon H. S., 1954. The economic theory of a common-property resource: The fishery, J. Polit. Eco., Vol. 62, pp. 124-142.

Guckenheimer J. and Holmes P., 1983. Nonlinear Oscillations, Dynamical Systems, and Bifurcations of Vector Fields, Springerverlag, New York.

Idels L. V. and Wang M., 2008. Harvesting fisheries management strategies with modified effort function, Int. Journal of Modelling, Identification and Control, Vol. 3, No. 1, pp. 83-87.

Kar T. K. and Pahari U. K., 2007. Modeling and analysis of a prey-predator system with stage-structure and harvesting, Nonlinear Anal., Vol. 8, No. 2, pp. 601-609.

Kar T. K. and Chakraborty K., 2010. Bioeconomic modelling of a prey predator system using differential algebraic equations, Int. Journal of Engg. Sci. and Tech., Vol. 2, No. 1, pp. 13-34.

Kot M., 2001. Elements of Mathematical Ecology, Cambridge.

Kumar S, Srivastava S. K. and Chingakham P., 2002. Hopf bifurcation and stability analysis in a harvested one-predator-two-prey 
model, Appl. Math. Comput., Vol. 129, No. 1, pp. 107-118.

Liu C., Zhang Q. and Zhang X., 2009. Dynamic analysis in a harvested differential-algebraic prey-predator model, Journal of Mechanics in Medicine and Biology, Vol. 9, No. 1, pp. 123-140.

Liu C., Zhang Q., Zhang Y. and Duan X., 2008. Bifurcation and control in a differential-algebraic harvested prey-predator model with stage structure for predator, Int. Journal of Bifurcation and Chaos, Vol. 18, No. 10, pp. 3159-3168.

Mazoudi E. E., Elalami N. and Mrabti M., 2008. Output feedback control for an exploited structured model of a fishing problem, Journal of Boilogical System, Vol. 16, No. 1, pp. 1-11.

Venkatasubramanian V., 1992. On a singular transformation for analyzing the global dynamics of a class of singular DAEs, Proc. Symp. Implicit and Nonlinear syst., pp. 364-371.

Venkatasubramanian V., Schättler H. and Zaborsky J., 1995. Local bifurcation and feasibility regions in differential-algebraic systems, IEEE Transcation and Automatic Control, Vol. 40, No. 12, pp. 1992-

2013.

Xiao D., Li W. and Han M., 2006. Dynamics in a ratio-dependent predator-prey model with predator harvesting, Math. Anal. Appl., Vol. 324, No. 1, pp. 14-29.

Zhang X., Zhang Q. and Zhang Y., 2009. Bifurcation of a class of singular biological economic models, Chaos Solitons \& Fractals, Vol. 40, No. 3, pp. 1309-1318.

Zhang Y. and Zhang Q., 2009. Optimal control of logistic bioeconomic model with singularity-induced bifurcation, Int. Journal of Information and System Sciences, Vol. 5, No. 3-4, pp. 369-379.

\section{Biographical notes}

Dr. Tapan Kumar Kar is an Associate Professor at the Department of Mathematics, Bengal Engineering and Science University, Shibpur, Howrah, India. His research interests include dynamical systems, stability and bifurcation theory, population dynamics, mathematical modeling in ecology and epidemiology, management and conservation of fisheries, bioeconomic modeling of renewable resources. He wrote around 66 academic papers on those topics. He also supervised several students of master and doctor degree.

Bapan Ghosh is currently a Ph. D scholar under the supervision of Dr. Tapan Kumar Kar in the Department of Mathematics, Bengal Engineering and Science University, Shibpur, Howrah, India. His research work is sponsored by Council of Scientific and Industrial Research (CSIR), Govt. of India. His research topics entitled as "Some model based study on the dynamics of exploited biological resources". Dynamical systems, stability and bifurcation theory, population dynamics, management and conservation of fisheries, bioeconomic modeling of renewable resources etc. are the main areas of interest. He obtained his M. Sc. (Applied Mathematics) degree in the year 2008 from University of Burdwan, Burdwan, India. His e-mail address is keshab_bg@yahoo.in.

Received July 2010

Accepted October 2010

Final acceptance in revised form October 2010 